\title{
Hemogasanalysis Point of Care (EPOC) in Pre- Hospital: The Importance of An Early Diagnosis of Silent Hypoxemia in a Context of Scarce Health- Care Resources.
}

Sara Montemerani ( $\sim$ sara.montemerani@gmail.com )

Azienda USL 8 Arezzo: Azienda USL Toscana sud est https://orcid.org/0000-0001-9208-5360

Asia Urbanelli

University of Siena Faculty of Medicine and Surgery: Universita degli Studi di Siena

Silvia Cini

Azienda USL Toscana sud est

Giovanni Sbrana

Azienda USL Toscana sud est

Thomas Tori

Azienda USL Toscana sud est

Massimo Mandò

Azienda USL Toscana sud est

Giovanni lannelli

Azienda USL Toscana sud est

Fernando Schiraldi

San Paolo Hospital: Ospedale San Paolo

\section{Research Article}

Keywords: Silent hypoxemia, Point-of-care tools (POCT), Arterial Blood Gas (ABG) analysis, COVID-19 disease, SARS-CoV-2, Emergency Medical Service (EMS).

Posted Date: August 24th, 2021

DOl: https://doi.org/10.21203/rs.3.rs-823204/v1

License: (9) (1) This work is licensed under a Creative Commons Attribution 4.0 International License. Read Full License 


\section{Abstract}

\section{Introduction}

Arterial blood gas ( $A B G)$ is a useful diagnostic test in the emergency setting. Thanks to the evolution of miniaturized technologies, it was possible to produce a Point of Care ABG analyzer that extended the use of blood gas analysis even in an out-of-hospital context. In the beginning of 2020, the Emergency Medical Service (EMS) of USL Toscana Sud-Est introduced a Point of Care ABG analyzer to characterize respiratory failure in pre-hospital. The onset of SARS-CoV-2 pandemic made the testing ground much more challenging. However, this situation highlighted the potential utility of the hand-held ABG analyzer for a better identification of silent hypoxemia in SARS-CoV-2 patients in pre-hospital setting.

\section{Methods}

\section{Primary endpoint}

Early identification of cases of silent hypoxemia in pre-hospital setting. In our retrospective observational analysis, we want to understand how many patients with silent hypoxemia the hand-held analyzer detect respect the standard measure of peripherical oxygen saturation (SpO2) at rest with pulse oximeter or respect the 6 minutes walking test.

\section{Design and setting}

We performed a retrospective descriptive analysis of 48 consecutive SARS-CoV-2 patients who activated the territorial Emergency Medical Service of Arezzo (USL Toscana Sud-Est). We included patients between October and November 2020. Age $<18$ and pregnancy were considered exclusion criteria. After the telephone triage, the operations center sent the ALS ambulance with a team made up of a physician and a nurse who performed a clinical evaluation of the patient and an arterial blood gas analysis directly at home. Arterial blood was collected from the patient's radial or brachial artery. ALS team directly visualized the result of the exam on EPOC.

\section{Results}

A total of 48 SARS-CoV-2 patients were collected, 28 men and 20 women, respectively. Nineteen of the total amounts of 48 SARS-CoV-2 patients had silent hypoxemia identified with the hospital ABG analyzer (gold standard). They didn't refer dyspnea or didn't show increased work of breathing during clinical evaluation. These patients had an arterial blood gas oxygen tension $\left(\mathrm{Pa}_{\mathrm{O} 2}\right)$ of less than $60 \mathrm{mmHg}$. EPOC identified 20 cases of silent hypoxemia instead of the 19 identified with the hospital blood gas analyzer (Sensibility 100\%, Specificity 97\%, VPP 95\%, VPN 100\% with 95\% Cl). The pulse oximeter detected 21 cases of silent hypoxemia (Sensibility $100 \%$, Specificity $94 \%$, VPP $89 \%$, VPN $100 \%$ with $95 \%$ Cl). The 6 minutes walking test detected only 11 of the 19 cases of silent hypoxemia because the test was aborted in 5 cases, and it was not performed in other 3 cases. 


\section{Conclusion}

From this first descriptive analysis, we conclude that hand-held blood gas analyzer is useful in the early identification of silent hypoxemia in COVID-19 patients. The EPOC system is a handheld and wireless solution that provides accurate results in less than one minute after sample introduction at the patient's side. The portability of this point-of-care tool make it potentially useful in pre-hospital clinical practice.

\section{Introduction}

Arterial blood gas ( $A B G)$ is a fundamental diagnostic test in many diseases. In the emergency setting, this examination is performed in a few seconds and, if correctly interpreted, provides useful information to the clinician and the staff who assist the patient. ABG is the main guide for the correct diagnosis of respiratory failure, subsequent monitoring, and assessment of the response to treatment. Thanks to the evolution of miniaturized technologies, it was possible to produce a handheld blood gas analyzer. This extended the use of blood gas analysis even in an out-of-hospital context, such as a patient's home, the ambulance, or the family doctor's office.

In the beginning of 2020, the Emergency Medical Service of USL Toscana Sud-Est introduced EPOC (Siemens Healthineers) by launching an experimental phase to evaluate its effectiveness, especially in the characterization of respiratory failure in pre-hospital. The onset of SARS-CoV-2 pandemic made the testing ground for this new Point of Care Test (POCT) much more challenging than expected. In the context of the public health crisis caused by SARS-Cov-2, the rapid transmission of the virus generated a large number of ill patients that exceeds the available health-care resources. We tried to understand whether the information provided by the addition of the blood gas analysis could be useful for a better identification of silent hypoxemia in SARS-CoV-2 patients.

\section{Methods}

\section{Primary endpoint}

Early identification of cases of silent hypoxemia in pre-hospital setting. Hypoxemia is defined as a decrease (below $60 \mathrm{mmHg}$ ) in the partial pressure of oxygen in arterial blood whereas hypoxia is defined by reduced level of tissue oxygenation.

Silent hypoxemia is a hypoxia that is tolerated by patients. Patients with silent hypoxemia do not experience tachypnoea or dyspnea but this condition is related to long-lasting damage of the lungs. In our retrospective observational analysis, we want to understand how many COVID-19 positive patients with silent hypoxemia the hand-held analyzer detect respect the standard measure of peripherical oxygen saturation $\left(\mathrm{SpO}_{2}\right)$ at rest with pulse oximeter or respect the 6 minutes walking test.

\section{Design and setting}


We performed a retrospective descriptive analysis of 48 consecutive SARS-CoV-2 patients who activated the territorial Emergency Medical Service of Arezzo (USL Toscana Sud-Est). We included patients between October and November 2020, when a second wave of COVID-19 affected Europe. Age < 18 and pregnancy were considered exclusion criteria. After the telephone triage, the operations center sent the ALS ambulance with a team made up of a physician and a nurse who performed a clinical evaluation of the patient and an arterial blood gas analysis directly at home (Fig. 1). Arterial blood was collected from the patient's radial or brachial artery. ALS team directly visualized the result of the exam on EPOC. The blood gas analytical data are subsequently stored on the database of the operations center. All the samples of the handheld system were reprocessed by the hospital blood gas analyzer to verify the accuracy of the results. No discrepancies were noted.

\section{Statistical analysis}

The data were analyzed using SPSS V.22 (SPSS, Chicago, Illinois, USA). Continuous variables are expressed as mean \pm standard deviation (SD) while categorical variables are expressed as number (percentage).

\section{Results}

A total of 48 SARS-CoV-2 patients were collected, 28 men and 20 women, respectively. The mean age of men was $66.3 \pm 17.5$, the mean age of women $69.1 \pm 20.1$. In Table 1 we have indicated the characteristics and the laboratory findings of the 48 patients collected (Table 1). All the 48 SARS-CoV-2 patients were evaluated by the ALS team at home. 36 COVID-19 positive patients were centralized for hospital care based on severe comorbidities (immunosuppression, severe COPD, heart disease, etc.) and/or the presence of symptoms such as severe dyspnea, altered vital signs, severe silent hypoxemia, and signs of hypoperfusion (confusion, falls, hypotension, cyanosis, chest pain). The remaining 12 patients continued home care with close monitoring. 
Table 1

Presenting patients' characteristics and laboratory findings

\begin{tabular}{|c|c|c|}
\hline & $\begin{array}{l}\text { n COVID-19 positive } \\
\text { hospitalized patients }\end{array}$ & $\begin{array}{l}\text { COVID-19 positive } \\
\text { home patients }\end{array}$ \\
\hline Blood gas characteristics in room air & Median (SD) & Median (SD) \\
\hline $\begin{array}{l}\text { Arterial partial pressure of oxygen } \\
(\mathrm{mmHg})\end{array}$ & $52.7(12.52)$ & $72.3(9.42)$ \\
\hline $\begin{array}{l}\text { Arterial partial pressure of carbon } \\
\text { dioxide }(\mathrm{mmHg})\end{array}$ & $37.53(7.46)$ & $33.65(5.89)$ \\
\hline $\mathrm{pH}$ & $7.44(0.08)$ & $7.48(0.06)$ \\
\hline $\mathrm{PaO} 2 / \mathrm{FiO} 2$ & $246.76(10.71)$ & $341.80(11.34)$ \\
\hline Lactate (mmol/L) & $2.2(0.3)$ & $1.6(0.4)$ \\
\hline Comorbidities & Total & Total \\
\hline Hypertension & 19 & 5 \\
\hline Diabetes & 9 & 3 \\
\hline Chronic kidney disease & 9 & 0 \\
\hline Cerebrovascular disease & 11 & 2 \\
\hline Cardiovascular disease & 15 & 3 \\
\hline Malignancy & 6 & 0 \\
\hline Autoimmune disease & 5 & 2 \\
\hline $\begin{array}{l}\text { Signs and symptoms at disease } \\
\text { onset }\end{array}$ & Total & Total \\
\hline Fever & 32 & 9 \\
\hline Cough & 27 & 10 \\
\hline Fatigue & 20 & 9 \\
\hline Myalgia & 17 & 8 \\
\hline Dyspnoea & 25 & 3 \\
\hline Diarrhoea & 11 & 2 \\
\hline Vomiting/Nausea & 4 & 0 \\
\hline Syncope & 8 & 0 \\
\hline Vital signs & Total & Total \\
\hline
\end{tabular}




\begin{tabular}{|c|c|c|c|}
\hline & $\mathbf{n}$ & $\begin{array}{l}\text { COVID-19 positive } \\
\text { hospitalized patients }\end{array}$ & $\begin{array}{l}\text { COVID- } 19 \text { positive } \\
\text { home patients }\end{array}$ \\
\hline$<24$ breaths per minute & & 19 & 12 \\
\hline $24-30$ breaths per minute & & 13 & 0 \\
\hline$>30$ breaths per minute & & 4 & 0 \\
\hline \multicolumn{4}{|c|}{ Systolic arterial pressure $(\mathrm{mmHg})$} \\
\hline$<90 \mathrm{mmHg}$ & & 3 & 0 \\
\hline $90-140 \mathrm{mmHg}$ & & 22 & 9 \\
\hline$>140 \mathrm{mmHg}$ & & 11 & 3 \\
\hline \multicolumn{4}{|l|}{ Heart rate $(\mathrm{bpm})$} \\
\hline$>100$ beats per minute & & 19 & 2 \\
\hline SD: Standard Deviation & & & \\
\hline
\end{tabular}

\section{Early detection of silent hypoxemia}

Several reports revealed that the prevalence of silent hypoxemia in COVID-19 patients ranges from 20 to $40 \%$, a similar percentage was also identified in our observational study. [1] Nineteen of the total amounts of 48 SARS-CoV-2 patients didn't refer dyspnea or didn't show increased work of breathing during clinical evaluation. These patients had an arterial blood gas oxygen tension $\left(\mathrm{Pa}_{\mathrm{O} 2}\right)$ of less than $60 \mathrm{mmHg}$ and didn't communicate to the ALS team any breathing difficulty. In addition, 14 of the 19 patients had arterial blood gas carbon dioxide tension $\left(\mathrm{Pa}_{\mathrm{CO}}\right)$ above $39 \mathrm{mmHg}$ (range 35-42), which would be expected to induce dyspnea; these patients had silent hypoxemia. These data were obtained from the hospital blood gas analyzer, that is considered the gold standard in the identification silent hypoxemia. EPOC identified 20 cases of silent hypoxemia instead of the 19 identified with the hospital blood gas analyzer (Sensibility $100 \%$, Specificity $97 \%$, VPP $95 \%$, VPN $100 \%$ with $95 \%$ Cl).

The pulse oximeter detected 21 cases of silent hypoxemia (Sensibility $100 \%$, Specificity $94 \%$, VPP $89 \%$, VPN $100 \%$ with $95 \% \mathrm{Cl}$ ). To limit the sources of error, all the measurements has been done indoor and with the patient at rest. Nail polish was removed, and cold extremities have been warmed before the measurement. ALS team considered the most common $\mathrm{SpO}_{2}$ measured value after an observation of 30-60 seconds.

The 6 minutes walking test detected only 11 of the 19 cases of silent hypoxemia. ALS team took a base line value of $\mathrm{SpO}_{2}$ with pulse oximeter at rest and after 6 minutes of walking. The test was considered positive when $\mathrm{SpO}_{2}$ resulted less than $94 \%$ or when there was an absolute drop of $\mathrm{SpO}_{2}$ by $3 \%$ or more from baseline. The test was aborted in 5 cases because patients felt unwell. In other 3 cases the test was 
not performed due to contraindications like hypomobility, valvular disease and unstable angina in anamnesis.

\section{Discussion}

One of the main goals in a general context of lack of ICU beds and overcrowded emergency departments, is to objectively evaluate patients and distinguish those who need hospital care from those who can be assisted at home (Fig. 2). However, in no case this should affect the right standard of care. Generally, severe dyspnea, altered vital signs and symptoms of hypoperfusion (confusion, falls, hypotension, cyanosis, chest pain) are related to patient's hospital admission. The problem related to patients with silent hypoxemia is that they cannot be strictly placed in these categories and in this way, we risk underestimating the disease. In pre-hospital, EPOC could help clinicians to early identify silent hypoxemia.

\section{Mechanism of silent hypoxemia}

As the literature data show, even minimal hypoxia can damage several organs. Oxygen limitation impairs mitochondrial respiratory chain and oxidative phosphorylation pathway. The impairment of oxidative phosphorylation pathway inhibits important enzymes' activities and leads to an energy-deprived condition. For this reason, it may be useful to combine patient's clinical evaluation with the use of a handheld blood gas analyzer that allows early identification of silent hypoxemia. [2] Respiratory distress may occur late in SARS-CoV-2 interstitial pneumonia. [3] This deviation from the typical picture of ARDS is probably due to a set of factors: the severity of the infection, the host's immune response, the patient's comorbidities, the level of response to hypoxia and the patient's respiratory functional reserve. [4] In COVID-19 patients, there are several mechanisms of hypoxia linked to the alteration of the ventilation/perfusion ratio (V/Q) that can be described. [5] First of all, it is possible to observe a deregulation of the normal hypoxia-induced vasoconstriction, due to hyperactivation of inflammatory mechanisms: vasoplegia causes a worsening of the V/Q ratio. [6] In addition, an activation of a cytokine storm and consequent coagulopathy can be observed, which favors thromboembolic events.

Microthrombosis in the pulmonary circulation can cause severe hypoxemia and deregulation of V/Q ratio. $[7,8]$

Unfortunately, it is also possible that SARS-CoV-2 has an idiosyncratic effect on the respiratory control system that reduces the efficacy of this physiologic mechanism. The angiotensin-converting enzyme 2 (ACE-2), which is the cell receptor of COVID-19, is expressed at the level of carotid oxygen chemoreceptors. Probably ACE-2 receptors play an important role in the depressed response to dyspnea in COVID-19 patients. [1]

Therefore, the early assessment of arterial blood gas parameters, such as $\mathrm{Pa}_{\mathrm{O} 2}, \mathrm{~Pa}_{\mathrm{CO} 2}$ and lactates and derived parameters, such as $\mathrm{Pa}_{\mathrm{O} 2} / \mathrm{Fi}_{\mathrm{O} 2}$ and the alveolar-arteriolar oxygen gradient, are essential for early diagnosis of silent hypoxemia, its severity and its classification. [9] 
Furthermore, from serial $A B G$, it is possible to highlight the increase in lactates, which is an early marker of respiratory muscle fatigue and diaphragmatic exhaustion. Our data shows that the median of lactates of COVID-19 hospitalized patients (median 2.2; SD 0.3) is lower than in COVID-19 home patients (median $1.6 ;$ SD 0.4). These results may be related to Gattinoni's hypothesis that states that lung compliance is at first greater and should determine a relatively lower Work of breathing (WOB) in the first stage of the disease $[10,11]$. Furthermore, having a baseline lactate value can be used to highlight its subsequent possible increase in a hospital setting. The increase of lactatemia during hospitalization may represent a useful monitoring tool of insufficient response to therapeutic interventions.

\section{Diagnosis of silent hypoxemia in COVID-19 patients}

Early detection of silent hypoxemia and start therapy to prevent further deterioration are crucial to minimize long-term effects as well as mortality rate in SARS-CoV-2 patients. [12]

In pre-hospital setting we have two widely used methods to identify silent hypoxemia: pulse oximetry and the 6 minutes walking test. The pulse oximetry is a common medical device used to measure peripherical oxygen saturation $\left(\mathrm{SpO}_{2}\right)$. Although it represents an accessible and hand-held device, it is important to underline that it has low accuracy when patient's $\mathrm{SpO}_{2}$ is lower than $90 \%$. In this case $\mathrm{SpO}_{2}$ can differ from true arterial saturation $\left(\mathrm{SaO}_{2}\right)$ measured with arterial blood gas analysis by as much as $4-7 \%$. [13] Furthermore, pulse oximetry does not provide any information about adequacy of ventilation, hemoglobin levels, electrolytes or cellular utilization of oxygen and these limitations should be considered when interpreting pulse oximetry findings. In our observational study we were able to find similar data (Fig. 3). Silent hypoxia is also detectable by 6 minutes walking test (peripherical oxygen saturation measured after 6 minutes of walk). In literature was underlined that 6 minutes walking test reported SpO2 levels significantly reduced in COVID-19 patients in comparison to the control group. [14] Despite that, this test has the disadvantage of not being feasible on all patients, for example in hypomobility syndromes or in patients with contraindications in anamnesis.

It was possible to add arterial blood gas analysis to the two methods previously listed thanks to the introduction of the hand-held ABG analyzer also in pre-hospital setting. This method has the advantage that it can be used to detect silent hypoxemia even in patients with reduced mobility and it has a greater accuracy in defining blood oxygenation values. Recent studies report that also $\mathrm{pH}$ level, bicarbonate and electrolyte levels may also be useful in silent hypoxia of COVID-19 patients. [15] Indeed, it has been reported that a lower level of $\mathrm{pH}$, sodium, calcium, and potassium are associated with increased disease severity and lower survival rates of COVID-19 patients. $[16,17]$ EPOC together with pulse oximeter and 6 minutes walking test could be precious tools for the early detection of silent hypoxia in SARS-CoV-2 positive patients in pre-hospital setting. [15] Therefore it can be an interesting and useful tool to be added to the instrumentation of the ALS team.

\section{Strengths and limitations of this study}


ABG was a highly validated tool in in-hospital setting. The study has the advantage of describing the use of the same tool for early identification of silent hypoxemia also in pre-hospital setting.

The study is the result of a first retrospective descriptive analysis of the data collected by EPOC during two months of the second wave of SARS-CoV-2 pandemic. It was a preliminary observational study with a small sample size. Further studies will be necessary to underline its usefulness in this context.

\section{Conclusion}

Our understanding of the strategies for the optimal management of SARS-CoV-2 pandemic continues to evolve. The approach described here is based upon our clinical experience in the pre-hospital setting.

From this first descriptive analysis, we conclude that hand-held blood gas analyzer is useful in the early identification of silent hypoxemia in COVID-19 patients. The EPOC system is a handheld and wireless solution that provides accurate results in less than one minute after sample introduction at the patient's side. The handling of this device, make it potentially useful in pre-hospital clinical practice as many other point-of-care tools (Fig. 4). Numerous other studies will be needed to understand how useful this technology can be in leaving the pandemic behind.

\section{List Of Abbreviations}

A-a gradient: Alveolar to arterial oxygen gradient

ABG: arterial blood gas

ARDS: acute respiratory distress syndrome

Cl: confidence interval

CPAP: continuous positive airway pressure

ED: emergency department

EMS: emergency medical service

ICU: intensive care unit

IOT: orotracheal intubation

$\mathrm{Pa}_{\mathrm{CO} 2}$ : carbon dioxide partial pressure

$\mathrm{Pa}_{\mathrm{O} 2}$ : arterial oxygen partial pressure

$\mathrm{Pa}_{\mathrm{O} 2} / \mathrm{Fi}_{\mathrm{O} 2}:$ arterial oxygen partial pressure/fractional inspired oxygen 
PEEP: positive end-expiratory pressure

POCT: point of care tool

$\mathrm{SaO}_{2}$ : arterial oxygen saturation

SD: standard deviation

$\mathrm{SpO}_{2}$ : peripherical oxygen saturation

VPN: negative predictive value

VPP: positive predictive value

V/Q: ventilation/perfusion ratio

WOB: work of breathing

\section{Declarations}

\section{Ethics Approval and Consent to participate}

This survey did not involve the collection or use of confidential or sensitive personal health information. Ethics approval was not required under the Geneva jurisdiction for reporting the data presented in this study. The present study was conducted in compliance with the Helsinki Declaration of 1975, as revised in October 2013.

\section{Consent for publication}

Not applicable.

\section{Availability of supporting data}

All data generated or analyzed during this study are included in this publication.

\section{Competing interests}

No one.

\section{Founding}

This work did not receive any specific grant from funding agencies in the public, commercial, or not-forprofit sectors.

\section{Authors' contributions}


S.M. and A.U. drafted the primary manuscript, contributed to the conceptualization of the study, and participated to the study selection/data collection; S.C. made the primary contributed to the conceptualization of the study; F.S., G.I. and G.S. revised both the primary manuscript and the study selection/data collection; G.S., T.T., M.M. G.I. and F.S. revised the manuscript and supervised all the work. All authors have read and agreed to the published version of the manuscript.

\section{Acknowledgements}

A thought to who have dedicated their experience and their work to the fight against the COVID-19 pandemic.

\section{References}

1. Tobin M, Laghi F, Jubran A. Why COVID-19 silent hypoxemia is baffling to physicians. Am J Respir Crit Care Med. 2020;202(3):356-60.

2. Rahman A, Tabassum T, Araf Y, et al. Silent hypoxia in COVID-19: pathomechanism and possible management strategy. Mol Biol Rep 2021; 48, 3863-3869.

3. WHO. Infection prevention and control during health care when novel coronavirus ( $\mathrm{nCoV}$ ) infection is suspected. Interim guidance. (WHO/COVID-19/IPC/v2020.1). Geneva, 2020.

4. Alhazzani W, Møller MH, Arabi YM, et al Surviving Sepsis Campaign: guidelines on the management of critically ill adults with Coronavirus Disease 2019 (COVID-19). Intensive Care Med (2020).

5. Dhont S, Derom E, Van Braeckel E, et al The pathophysiology of 'happy' hypoxemia in COVID-19. Respir Res 21, 198 (2020).

6. Lang M, Som A, Mendoza DP, et al. Hypoxaemia related to COVID-19: vascular and perfusion abnormalities on dual-energy CT. Lancet Infect Dis. 2020;20(12):1365-1366.

7. Ackermann M, Verleden SE, Kuehnel M, et al. Pulmonary vascular endothelialitis, thrombosis, and angiogenesis in Covid-19. N Engl J Med 2020 May 21.

8. Wang T, Chen R, Liu C, et al. Attention should be paid to venous thromboembolism in patients with severe novel coronavirus pneumonia. Lancet Haematol 2020; 7:362-3.

9. Scott S, Antonaglia V, Guiotto G, et al. Two-hour lactate clearance predicts negative outcome in patients with cardiorespiratory insufficiency. Crit Care Res Pract. 2010; 2010:917053.

10. Gattinoni L, Coppola S, Cressoni M, et al. COVID-19 Does Not Lead to a "Typical" Acute Respiratory Distress Syndrome. American journal of respiratory and critical care medicine. 2020; 201(10), 12991300.

11. Gattinoni L, Chiumello D, Caironi P, et al. COVID-19 pneumonia: different respiratory treatments for different phenotypes? Intensive Care Med. 2020 Jun;46(6):1099-1102.

12. Teo J. Early Detection of Silent Hypoxia in Covid-19 Pneumonia Using Smartphone Pulse Oximetry. J Med Syst. 2020;44(8):134. 
13. Quaresima V, Ferrari M. COVID-19: efficacy of prehospital pulse oximetry for early detection of silent hypoxemia. Crit Care. 2020;24(1):501.

14. RE: COVID-19, on the edge of hypoxia [Internet]. Science. 2020 [cited 2 September 2020]. Available from: https://science.sciencemag.org/content/re-covid-19-edge-hypoxia.

15. Elezagic D, Johannis W, Burst V, et al. Venous blood gas analysis in patients with COVID-19 symptoms in the early assessment of virus positivity. LaboratoriumsMedizin. 2020;0(0).

16. Skevaki C, Fragkou P, Cheng $C$, et al. Laboratory characteristics of patients infected with the novel SARS-CoV-2 virus. J Infect. 2020;81(2):205-212.

17. Lippi G, South A, Henry B. Electrolyte imbalances in patients with severe coronavirus disease 2019 (COVID-19) Annals ClinBiochem Intern J Lab Med. 2020;57(3):262-265.

\section{Figures}

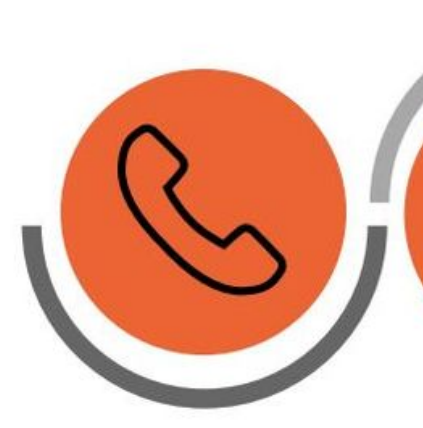

Emergency response system activation

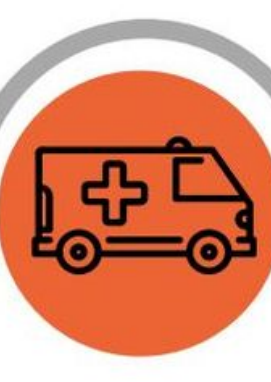

EMS response

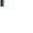

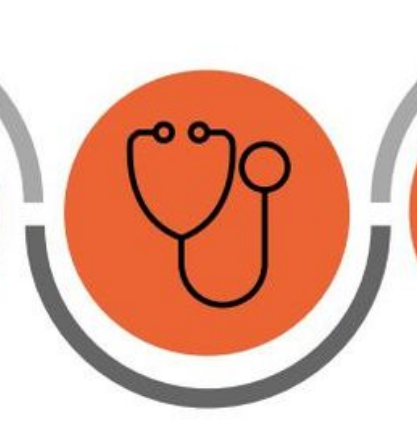

Early EMS clinical evaluation.

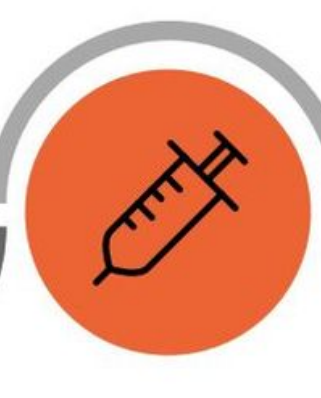

Point of care ABG

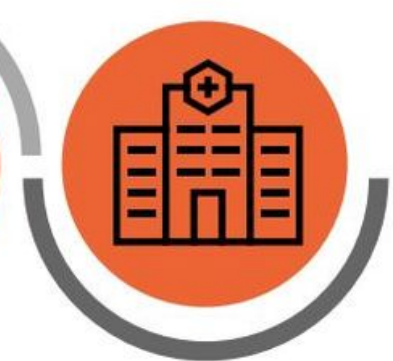

Care setting

\section{Figure 1}

Algorithm of Emergency Medical Service activation. 
I T A L Y : C O V I D-19

CASES C U R VES

$\mathrm{New}$ Infected

Decessed

Recovered

Active

\section{C U P A T I E N T S}

Number of COVID 19 positive patients in Intensive Care Units in Italy. The orange line show the first alert threshold, the red line the second.
4000000

3200000

2400000

1600000

800000

A

5000

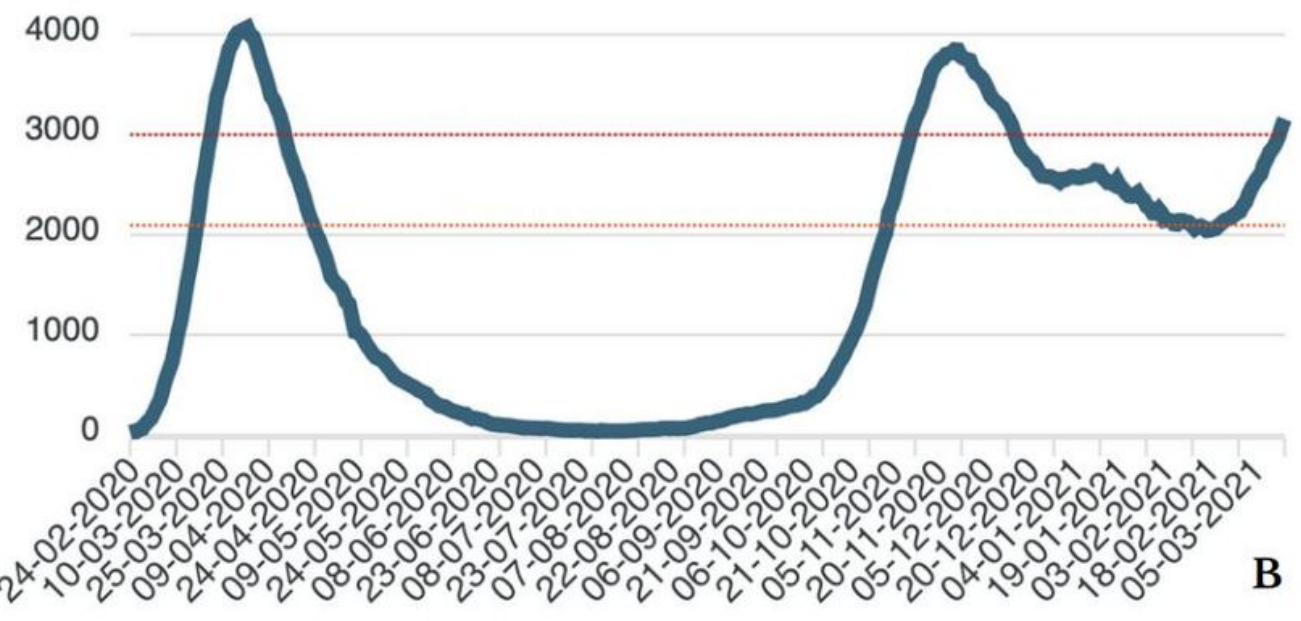

\section{Figure 2}

Italy: COVID-19 cases curves and ICU patients. 


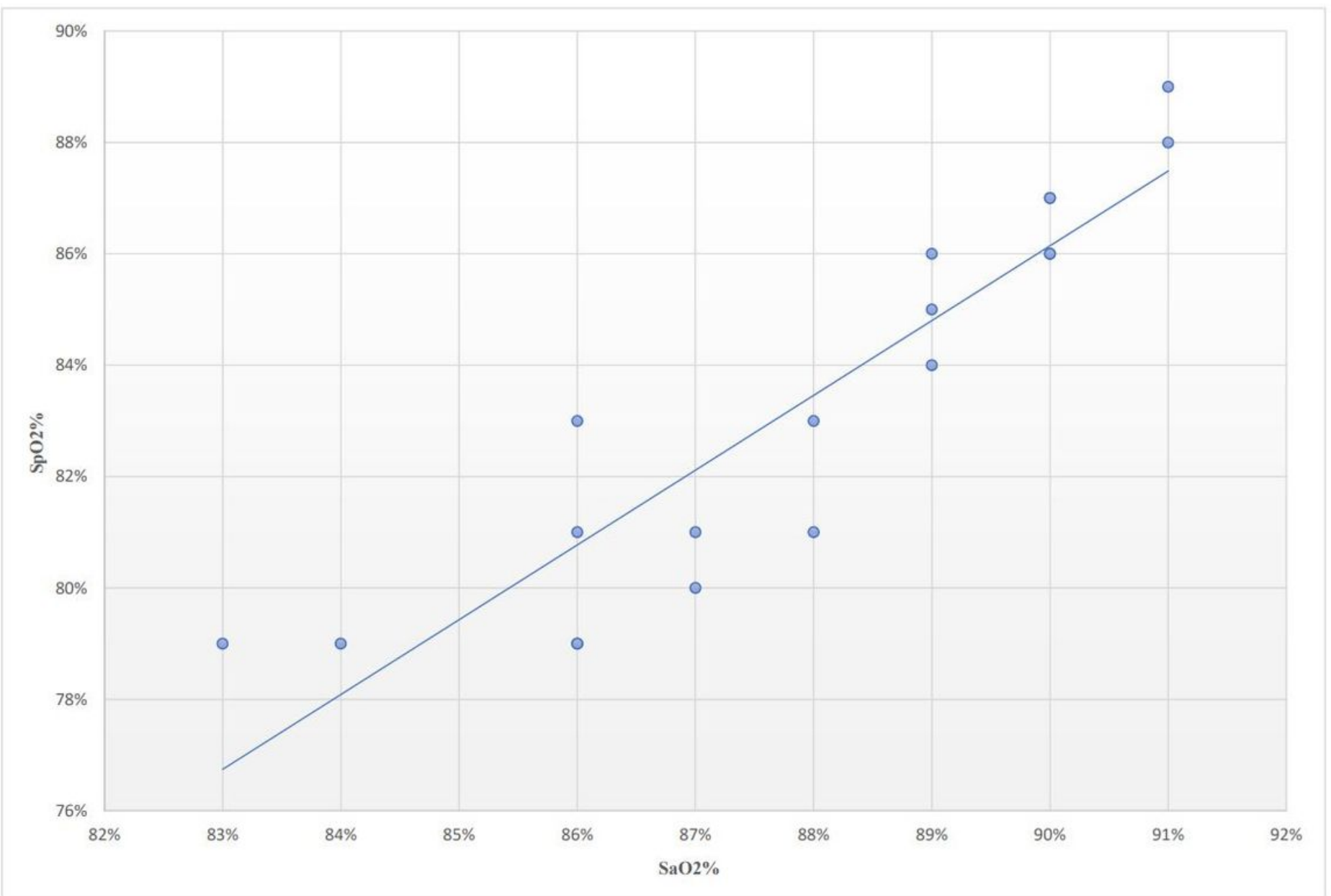

\section{Figure 3}

Scatterplot of the relationship between estimated oxygen saturation from pulse oximetry (SpO2) and $\mathrm{SaO} 2$ from hand-held blood gas analysis in 19 COVID-19 patients with silent hypoxemia. Each subject is represented by a point. The solid line is the regression line. 


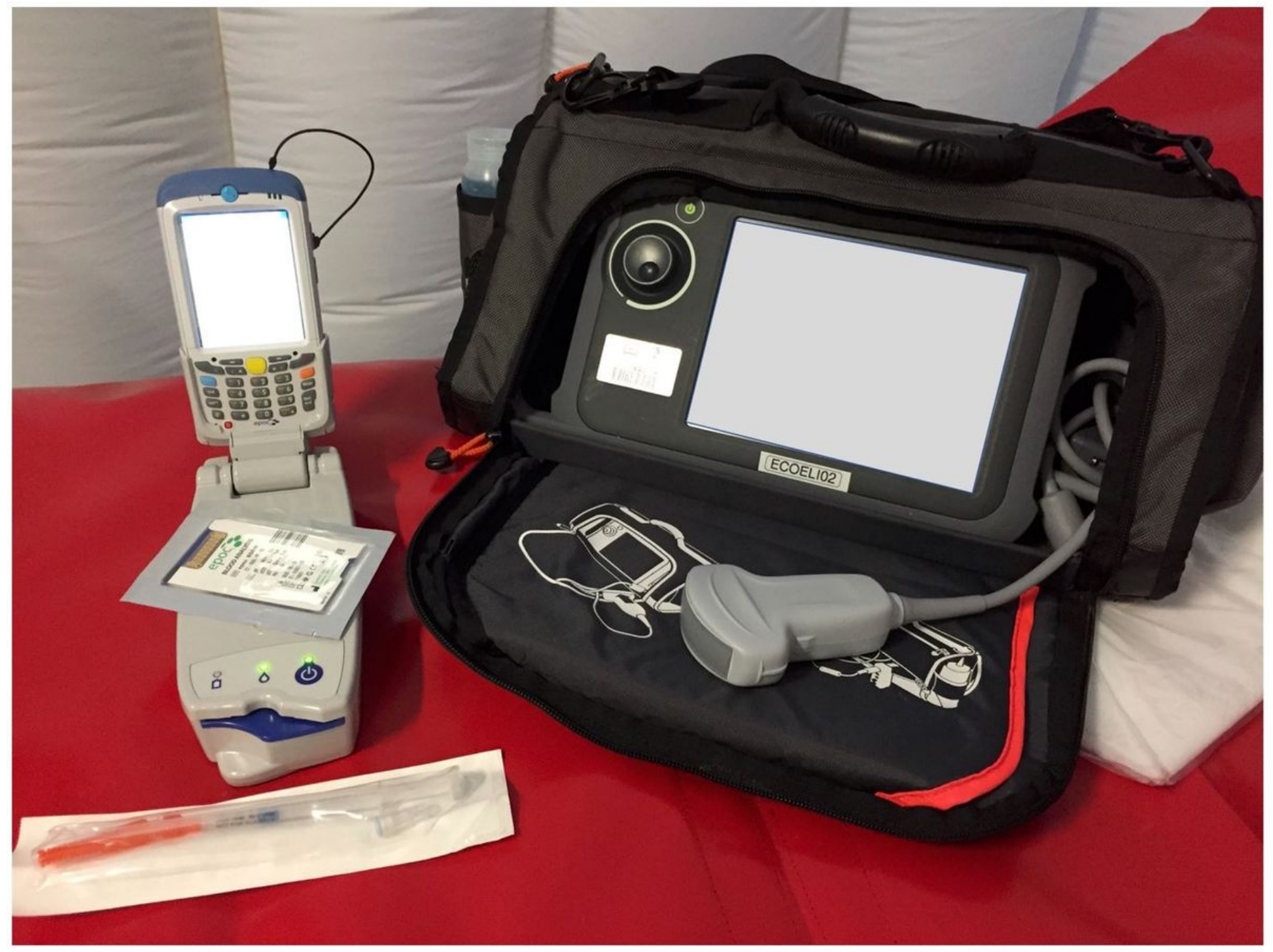

Figure 4

Point of care tools: hand-held blood gas analyzer and portable ultrasound system. 